\title{
Defining remission: proposed criteria and rationale for consensus Nancy Andreasen*
}

\author{
Address: University of Iowa, USA \\ * Corresponding author
}

\author{
from International Society on Brain and Behaviour: 2nd International Congress on Brain and Behaviour \\ Thessaloniki, Greece. 17-20 November 2005 \\ Published: 28 February 2006 \\ Annals of General Psychiatry 2006, 5(Suppl I):S29 doi:10.1 I86/1744-859X-5-SI-S29
}

New advances in the understanding of schizophrenia etiology, course, and treatment have increased interest on the part of patients, families, advocates, and professionals in the development of consensus-defined standards for clinical status and improvement, including illness remission and recovery. As demonstrated in the area of mood disorders, such standards provide greater clarity around treatment goals, as well as an improved framework for the design and comparison of investigational trials and the subsequent evaluation of interventional effectiveness. Unlike mood disorders, however, the novel application of the concept of standard outcome criteria to schizophrenia must reflect the wide heterogeneity of chronic psychotic illness course and outcome, as well as the variable effects of different treatments on schizophrenia symptomatology. As an initial step in developing operational criteria, an expert Working Group reviewed available definitions and assessment instruments to provide a conceptual framework for symptomatic, functional and cognitive domains in schizophrenia as they relate to remission of illness. The first consensus-based operational criteria for symptomatic remission in schizophrenia is based on distinct thresholds for reaching and maintaining improvement, as opposed to change criteria, allowing for alignment with traditional concepts of remission in both psychiatric and non-psychiatric illness. This innovative approach for standardizing the definition for outcome in schizophrenia also calls for further examination of its validity and utility, as well as the potential for future refinement, particularly in relation to the growing characterization of cognitive dysfunction in psychotic illness. These criteria should facilitate research and support a positive, longer-term approach regarding outcome for patients with schizophrenia. 